\title{
High-Throughput Topographic, Mechanical, and Biological Screening of Multilayer Films Containing Mussel-Inspired Biopolymers
}

\author{
Ana I. Neto, Natália L. Vasconcelos, Sara M. Oliveira, Daniel Ruiz-Molina, \\ and João F. Mano*
}

\begin{abstract}
A high-content screening method to characterize multifunctional multilayer films that combine mechanical adhesion and favorable biological response is reported. Distinct combinations of nanostructured films are produced using layer-by-layer methodology and their morphological, physicochemical, and biological properties are analyzed in a single microarray chip. Inspired by the composition of the adhesive proteins in mussels, thin films containing dopamine-modified hyaluronic acid are studied. Flat biomimetic superhydrophobic patterned chips produced by a bench-top methodology are used for the buildup of arrays of multilayer films. The wettability contrasts imprinted onto the chips are allowed to produce individual, position controlled, multilayer films in the wettable regions. The flat configuration of the chip permits to perform a series of nondestructive measurements directly on the individual spots. In situ adhesion properties are directly measured in each spot, showing that nanostructured films richer in dopamine promote the adhesion. In vitro tests show an enhanced cell adhesion for the films with more catechol groups. The advantages presented by this platform include ability to control the uniformity and size of the multilayers films, its suitability to be used as a new low cost toolbox and for high-content cellular screening, and capability for monitoring in situ a variety of distinct material properties.
\end{abstract}

\section{Introduction}

In the field of nanotechnology, many efforts have been made to control shape, size distribution, and surface chemistry of nanostructures. $^{[1]}$ Specifically, adsorption of biopolymers in defined spatial surface arrangements offers a promising skill for the generation of innovative nanostructures, suitable for a broad range of applications in the fields of biomedical materials, tissue engineering, and diagnosis. So, development of novel tailored simple and versatile strategies for throughput biopolymer surface modification and its corresponding characterization have become a challenging area of continuous research over the years. ${ }^{[2,3]}$ Among the different approaches so far reported, ${ }^{[4]}$ the layer-bylayer (LbL) deposition technique represents an effective and reproducible method, with a wide range of advantages, particularly in the biomedical field, as detailed next: (i) this technique can be performed in virtually any kind of substrate (planar, porous, colloidal, among others) and different surface chemistries, (ii) the film organization can be spatiotemporal controlled, and (iii) the assembly can be performed under "mild" conditions with a large variety of inexpensive materials compatible with physiological media, permitting also the integration of large amounts of bioactive molecules and extracellular matrix components in the films. ${ }^{[5,6]}$ Moreover, the final biological, physicochemical, and mechanical properties of the multilayered films can be finely tuned by controlling multiple factors, such as the nature of the inserted components, their distribution along the thickness of the film (e.g., the nature of the top layer), the number of layers, the introduction of any additional molecules, or the post-treatment of the film (e.g., crosslinking). However, even successful as previously described, this technique most often requires expensive and time-consuming methodologies that represent a great shortcoming limiting its applicability. Hence, novel experimental approaches that allow for a rapid, simple, and accurate screening of multilayer arrays obtained from the large portfolio of available biopolymers, processing conditions and combinations of them, are needed.

DOI: 10.1002/adfm.201505047 
High-throughput screening (HTS) technologies represent an excellent tool with this aim. ${ }^{[7-11]}$ Distinct HTS procedures have already been used for the production of LbL constructs based on: selective deposition, ${ }^{[12]}$ microfluidics, ${ }^{[13,14]}$ disruption of hydrogen-bonded multilayers, ${ }^{[15,16]}$ and photoreactive multilayers. $^{[17]}$ For instance, Barret et al. reported a combinatorial analysis of 5000 individual synthetic polyelectrolyte multilayers with modulated 2D physical properties and thicknesses gradients simply by rotating the substrate along the deposition process. ${ }^{[18]}$ Hammond and co-workers, ${ }^{[19]}$ used a capillary flow technique to produce gradients, based on patterned isolated spots of a microfluidic chip with LbL thin film constructs. However, in spite of these pioneering examples, the preparation of discrete and nanostructured multilayered LbL films on a single substrate that allow for a fast HTS screening has not yet being reported.

Herein, we fill this gap with the use of a superhydrophobic, flexible, and flat platform decorated with patterned arrays of wettable regions acting as liquid reservoirs, where several combinations of nanostructured multilayer films can be obtained with a full control in space and time. A schematic representation of the patterned chip along with the fabrication procedure and the corresponding characterization of the arrays are shown in Figure 1. This new setup can allow for the first time to individually analyze several distinct small-sized multilayer films within a single chip, where the number of layers, crosslinking, protein adsorption, and nature of terminal layers are systematically tuned. As a proof-of-concept to demonstrate the feasibility of our approach, we have studied how the functionalization of polyelectrolytes with different amounts of dopamine (DN) affect the surface properties (morphology and wettability), the mechanical behavior and cell adhesion performance of the resulting LbL films through a combinatorial analysis of different experiments. The conjugation of polymers with DN, inspired by the structure and composition of an unusual amino acid presented in mussels protein (3,4-dihydroxyphenylalanine, DOPA), ${ }^{[20]}$ has already been shown to improve the adhesive properties of the coatings. ${ }^{[21-24]}$ For instance, multilayered films of hyaluronic acid (HA) and DN showed applicability for tissue engineering and biomedical applications. ${ }^{[25]}$ However, and in spite of its clear interest, there was a lack of detailed and systematic studies that allow for a proper understanding, and therefore optimization, of films containing DN. This fact has been achieved here thanks to our new high-throughput chip where several different combinations of HA-DN and chitosan (CHT) are studied and compared between them (CHT and alginate (ALG) are also studied for comparison purposes).

\section{Results and Discussion}

\subsection{Study of the LbL Multilayer Films Formation}

Initially, LbL films containing the targeted polymeric combinations to be deposited on the chip were first obtained as bulk

\section{a) Patterned SH surface with transparent spots preparation}

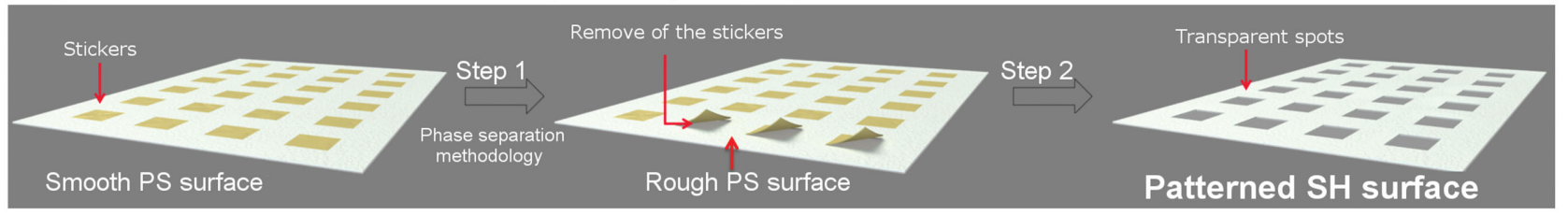

b) LbL build-up on patterned SH chip

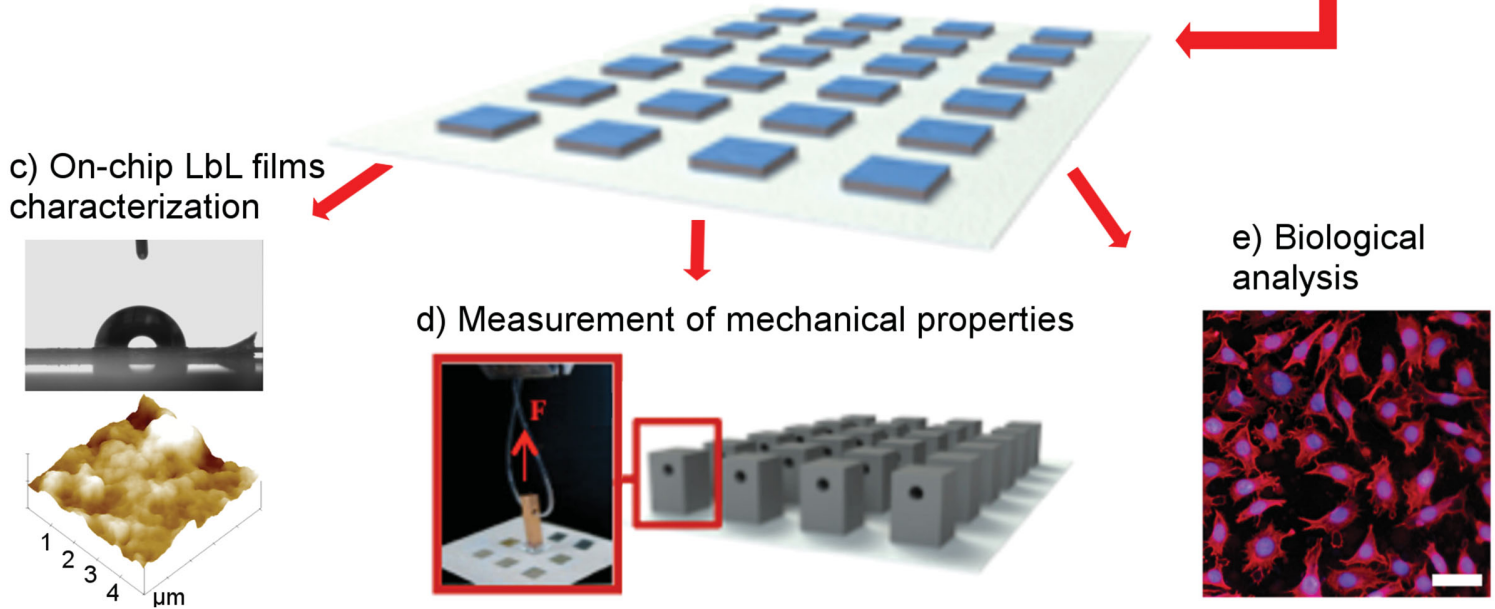

Figure 1. a) Preparation of superhydrophobic (SH) chips patterned with arrays of wettable regions prepared as detailed next: Initially an array of stickers in a polystyrene untreated film is formed; step 1 refers to the same array upon inducing a roughness increase with an additional in situ deposition of polystyrene, and therefore inducing an increase of the $\mathrm{SH}$ character at the macroscopic level. In step 2, wettable areas are obtained by removing the stickers. b) Build-up of layer-by-layer films using different combinations of polyelectrolytes solutions in the wettable spots surrounded by SH domains. Afterward, c) surface characterization, d) mechanical adhesion properties, and e) biological response of the multilayer films are assessed directly on the different spots. Scale bar: $50 \mu \mathrm{m}$. 
surfaces and the film growth studied to get more insight into their formation. The choice of polycationic polysaccharides is very limited and among them, CHT the de-acetylated form of chitin, was selected because being far the most widespread biopolymer used in polyelectrolyte multilayer films due to its numerous interesting properties, ${ }^{[26]}$ including biodegradability, biocompatibility, and antibacterial properties. As a negatively charged polymer, we have used HA. This is a polysaccharide containing alternating $N$-acetyl-D-glucosamine and D-glucuronic acid monosaccharide units. As an integral component of extracellular matrix, ${ }^{[27]} \mathrm{HA}$ is an attractive building block for new biocompatible and biodegradable materials that can be used for drug delivery and tissue engineering. ${ }^{[28,29]}$ Moreover, HA is well known to complex with CHT; for instance, LbL deposition of a combination of both polymers has already been used to repair damaged blood vessels. ${ }^{[30]}$ Finally, to bring more insights upon catechol substitution, two conjugated biopolymers based on DN-modified hyaluronic acid (HA-DN and HA-4DN), that differ in the amount of DN conjugated, the later having a fourfold excess of the dopamine used in the reaction, have also been studied. The real degree of dopamine substitution in each case was obtained by following the UV excitation band centered at $280 \mathrm{~nm}$ and upon comparison with a standard curve obtained for distinct concentrations of free dopamine in solution (for more details see the Experimental Section). ${ }^{[31]}$ UV-vis spectra of the different polymers used in this work are shown in Figure 2. Using the measured absorbance, the obtained concentration of dopamine units in both conjugates was $11 \%$ and $15 \%$ for HA-DN and HA-4DN, respectively.

Once the selection of functional polymers was done, the progress of LbL assembly was monitored by quartz crystal microbalance with dissipation (QCM-D), as shown in Figure $2 \mathrm{~b}$. This technique is able to detect mass changes in the order of nanograms per square centimeter while it measures the viscoelastic properties of the surface. ${ }^{[32]}$ During QCM-D measurements, a favorable and successful construction is identified by a decrease in $\Delta f$ caused by a mass adsorption at the top of the sensor. The $\Delta f / v$ (where $v$ is the overtone) decreased upon flushing the substrate with the sequence of polyelectrolytes, due to the deposition of the new polymer layer on the surface of the crystal. At the same time, monitoring of $\Delta D$ gives an indication of the film viscoelastic properties. An increase in $\Delta D$ represents a shift towards a film with a higher viscous component and damping properties that characterize a softer and hydrated film that is deposited over the metallic sensor.

Figure $2 \mathrm{~b}$ shows the normalized frequency, $\Delta f / v$ and dissipation variation, $\Delta D$, for the 5 th overtone $(25 \mathrm{MHz})$ of the three targeted polymeric multilayers (five layers) grown on goldcoated quartz sensors: (CHT/HA-DN $)_{5},(\mathrm{CHT} / \mathrm{HA}-4 \mathrm{DN})_{5}$, and $(\mathrm{CHT} / \mathrm{HA})_{5}$. In addition to the different $\mathrm{CHT} / \mathrm{HA}$ mixtures, a negatively charged polymer such as ALG was also used for comparison purposes. ${ }^{[33-35]}$ Each solution adsorption step corresponds to a decrease of $\Delta f$ with each CHT, HA-4DN, HA-DN, HA or ALG injection and a subsequent increase in $\Delta f / v$ due to desorption of a small fraction of free polyelectrolyte during the rising step. In parallel, an increase of $\Delta D$ is found, meaning that the materials deposited onto the crystal surface exhibit viscoelastic properties. Typically, a lower $\Delta D$ variation indicates that the film layer becomes denser and a strong asymmetric growth
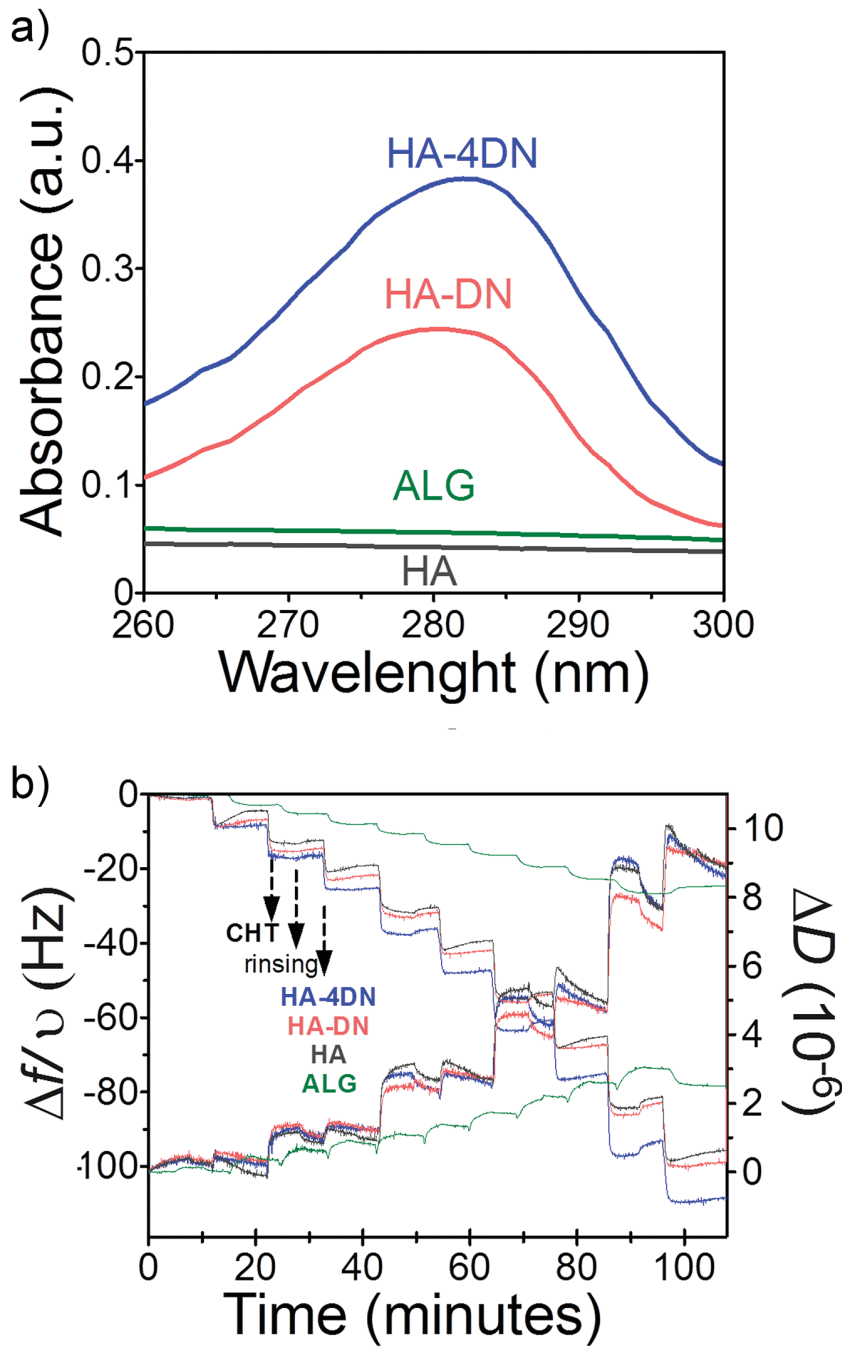

Figure 2. a) UV-vis spectra of the conjugates (HA-DN and HA-4DN) and the controls (HA and ALG), b) QCM-D monitoring of the LbL construction of $\mathrm{CHT}$ with the conjugates (HA-DN, HA-4DN) and $\mathrm{CHT}$ with the controls (HA and ALG) up to five deposition bilayers in $0.15 \mathrm{M} \mathrm{of} \mathrm{NaCl}$, maintaining the $\mathrm{pH}$ at 5.5 .

occurs, displaying a linear growth law. Parameters, such as the chemical nature of the polymer pair or the concentration of salt added to solutions used for deposition and/or rinsing have also a strong influence on the nature of the film formed. ${ }^{[36]}$ We also revealed that multilayer films containing catechol groups exhibit a linear film growth, thus giving rise to more rigid thin films. The results evidence the assembly of chitosan with the conjugates within the microarrays as those used as control as already reported..$^{[37-41]}$ Build-up of the multilayer film using the new conjugate (HA-4DN) has never been reported before.

\subsection{Multichip Fabrication and Topographic Characterization}

The working principle behind the platform developed in this work consists of a polystyrene (PS) superhydrophobic ( $\mathrm{SH}$ ) surface patterned with wettable regions produced by a benchtop methodology. Briefly, square-shaped stickers were attached 
to polystyrene film, and afterward the resulting surface treated and coated with rough polystyrene by a phase-separation method. ${ }^{[42]}$ Afterward, the stickers were totally removed and the whole surface washed with pure ethanol in order to avoid possible traces of the adhesive (Figure 1a). While the dots obtained upon removal of the stickers exhibit a proper wettability, macroscopically the film still retains its $\mathrm{SH}$ behavior thanks to the peculiar lotus-like features including micro- and nanoroughness and low surface energy. ${ }^{[43]}$ By using such wettability contrast, in each spot, sequentially deposition of droplets with different polyelectrolytes, combined with washing solutions, were performed to assemble the multilayers (Figure 1b). An array of 48 spots, combining 5, 10, or 15 bilayers prepared from a library of five polyelectrolytes (four distinct pairs), was designed with four replicates per combination to obtain statistical information.

Representative scanning electron microscopy (SEM) images shown in Figure 3 revealed the final surface topography of the polymeric arrays obtained directly from the developed substrate. A more quantitative analysis was done by atomic force microscopy (AFM) (see also Figure 3). The root mean square $\left(R_{\mathrm{q}}\right)$ and average roughness $\left(R_{\mathrm{a}}\right)$ obtained by AFM topographical studies are summarized in Table 1 . As can be seen there, all the surfaces exhibit a roughness that increases upon increasing the number of deposited layers, in agreement with the work reported by Lee and co-workers. ${ }^{[44]}$

Diffusion processes take place during the assembly of the CHT/HA and CHT/ALG systems, which growth in an exponential regime. So, the final thickness of these films is mainly related to the ability of CHT to diffuse "in" and "out" in the whole film during each deposition step and also to the nondesorption of the CHT/HA and CHT/ALG layers during the rinsing steps. The results suggest that the interface of CHT/HA and CHT/ALG is getting rougher and the surface area is increasing due to the fact that the surface becomes hydrated.
Hydration is a critical parameter in polyelectrolyte multilayer films, impacting film thickness, mechanical/viscoelastic properties, swelling ability, transport properties, and molecular mobility. ${ }^{[45]}$

Related with the surface topography, the wettability of the films was also evaluated in situ by water contact angle (WCA) measurements and the results included in Table 1 . The volume of the water droplets was small enough so that the WCA measurements could be performed in the individual spots. The changes in the water contact angle and topography of the glass control provided additional evidence of the presence of the coatings. As a main tendency, except for the CHT/ALG combination, it was found that the WCA values increase upon increasing the number of deposited layers. Moreover, a gradual increase of the catechol content is translated into a small increase of the WCA; for instance, the CHT/HA-4DN value for 15 layers is around $89^{\circ}$, which is slightly larger than $\mathrm{CHT} /$ HA-DN films for the same number of layers (around $85^{\circ}$ ) and $\mathrm{CHT} / \mathrm{HA}$ (around $82^{\circ}$ ).

\subsection{In Situ Mechanical Test}

Methods for screening adhesiveness rely on the measurement of either tensile or shear strengths; according to Thirawong et al. ${ }^{[46]}$ The first test usually uses perpendicular forces necessary to separate two surfaces in contact, while the second determines the force that causes an adhesive to slide on a surface in direction parallel to the plane of contact. In the present study, tensile strengths were chosen and measured for multilayer films with 5, 10, and 15 bilayers built-up in the wettable spots of the SH chip. For this, iron pillars containing the surface base with the same size and shape of the spots, were put in contact with the top of the films. The mechanical properties of the films were then assessed after $24 \mathrm{~h}$ of contact using the lap adhesive

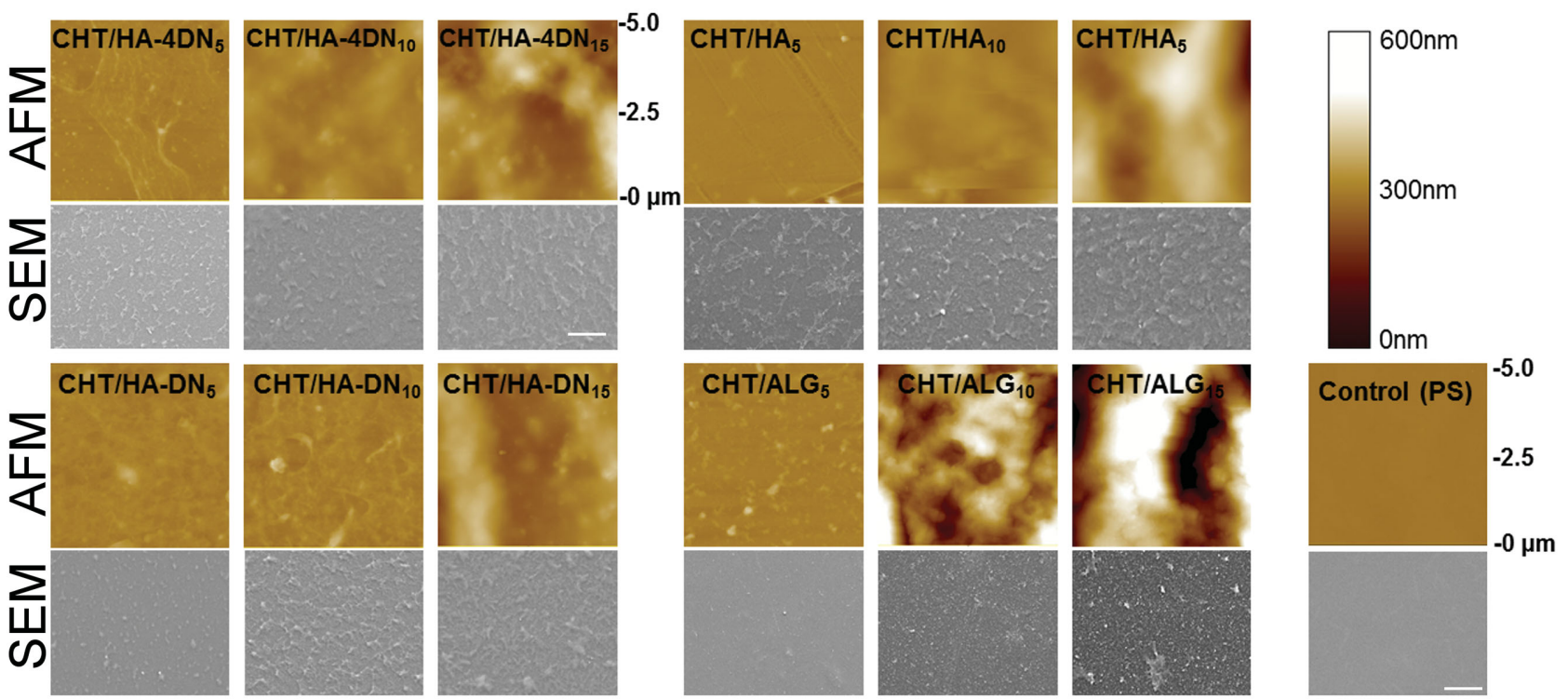

Figure 3. Representative AFM and SEM images of CHT/HA-DN, CHT/HA-4DN, CHT/HA, and CHT/ALG films with 5, 10, and 15 bilayers, assembled in the wettable spots of the patterned SH surface. The images of the control (polystyrene surface) are also shown for comparison purposes. Scale bar of SEM images: $5 \mu \mathrm{m}$. 
www.MaterialsViews.com

Table 1. Root mean square $\left(R_{\mathrm{g}}\right)$, average roughness $\left(R_{\mathrm{a}}\right)$, and water contact angle (WCA, $3 \mu \mathrm{L}$ drop volume) of the studied multilayered surfaces $(n=5)$ taken directly from the developed chips.

\begin{tabular}{|c|c|c|c|c|c|c|c|}
\hline \multirow[b]{2}{*}{ Number of layers } & \multicolumn{4}{|c|}{$\mathrm{CHT} / \mathrm{HA}-\mathrm{DN}$} & \multicolumn{3}{|c|}{$\mathrm{CHT} / \mathrm{HA}-4 \mathrm{DN}$} \\
\hline & \multicolumn{2}{|c|}{5} & 10 & 15 & 5 & 10 & 15 \\
\hline $\mathrm{R}_{\mathrm{q}}[\mathrm{nm}]$ & \multicolumn{2}{|c|}{$10.2 \pm 0.7$} & $10.8 \pm 1.7$ & $41.2 \pm 5.6$ & $9.1 \pm 0.4$ & $11.2 \pm 2.2$ & $47.9 \pm 6.1$ \\
\hline $\mathrm{R}_{\mathrm{a}}[\mathrm{nm}]$ & \multicolumn{2}{|c|}{$7.9 \pm 0.6$} & $7.6 \pm 1.1$ & $32.9 \pm 4.6$ & $6.9 \pm 0.7$ & $7.1 \pm 0.2$ & $39.9 \pm 4.0$ \\
\hline \multirow[t]{2}{*}{ WCA [ [ $]$} & \multicolumn{2}{|c|}{$66.9 \pm 3.7$} & $78.7 \pm 4.1$ & $84.9 \pm 2.1$ & $71.4 \pm 4.9$ & $79.5 \pm 2.5$ & $89.2 \pm 3.7$ \\
\hline & \multicolumn{3}{|c|}{$\mathrm{CHT} / \mathrm{HA}$} & \multicolumn{3}{|c|}{$\mathrm{CHT} / \mathrm{ALG}$} & Control (PS) \\
\hline Number of layers & 5 & 10 & 15 & 5 & 10 & 15 & Number of layers \\
\hline $\mathrm{R}_{\mathrm{q}}[\mathrm{nm}]$ & $6.4 \pm 1.2$ & $13 \pm 0.9$ & $65.9 \pm 1.8$ & $13.6 \pm 3.8$ & $87.8 \pm 21.2$ & $156.5 \pm 13.3$ & $1.7 \pm 0.2$ \\
\hline $\mathrm{R}_{\mathrm{a}}[\mathrm{nm}]$ & $3.7 \pm 0.2$ & $10.4 \pm 0.9$ & $52.9 \pm 2.4$ & $7.9 \pm 1.8$ & $69.6 \pm 15.9$ & $122.6 \pm 19.4$ & $1.4 \pm 0.1$ \\
\hline WCA $\left[{ }^{\circ}\right]$ & $61.7 \pm 1.3$ & $77.6 \pm 1.6$ & $82 \pm 3.4$ & $66.4 \pm 4.1$ & $53.5 \pm 0.7$ & $18.1 \pm 1.6$ & $92.2 \pm 4.1$ \\
\hline
\end{tabular}

strength test according to the standard protocol ASTM D1002 (see Video 1 in the Supporting Information for a better understanding of this procedure). For these measurements, the pillars were pulled until the total detachment from the base of the chip is achieved. Representative load-extension curves for the detachment of the pillars from the coatings are presented in Figure 4a.

As can be seen there, the elongation modulus of the distinct multilayer films is similar, but the final displacement of

a)
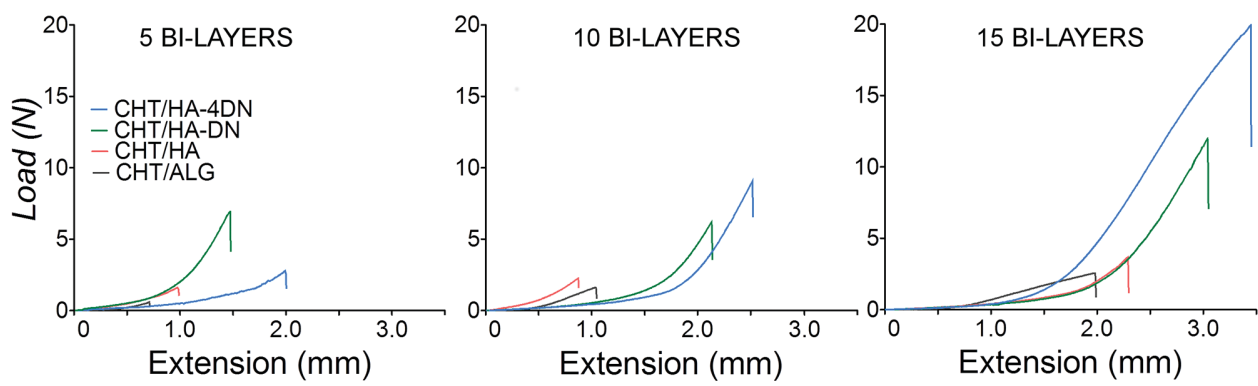

b)

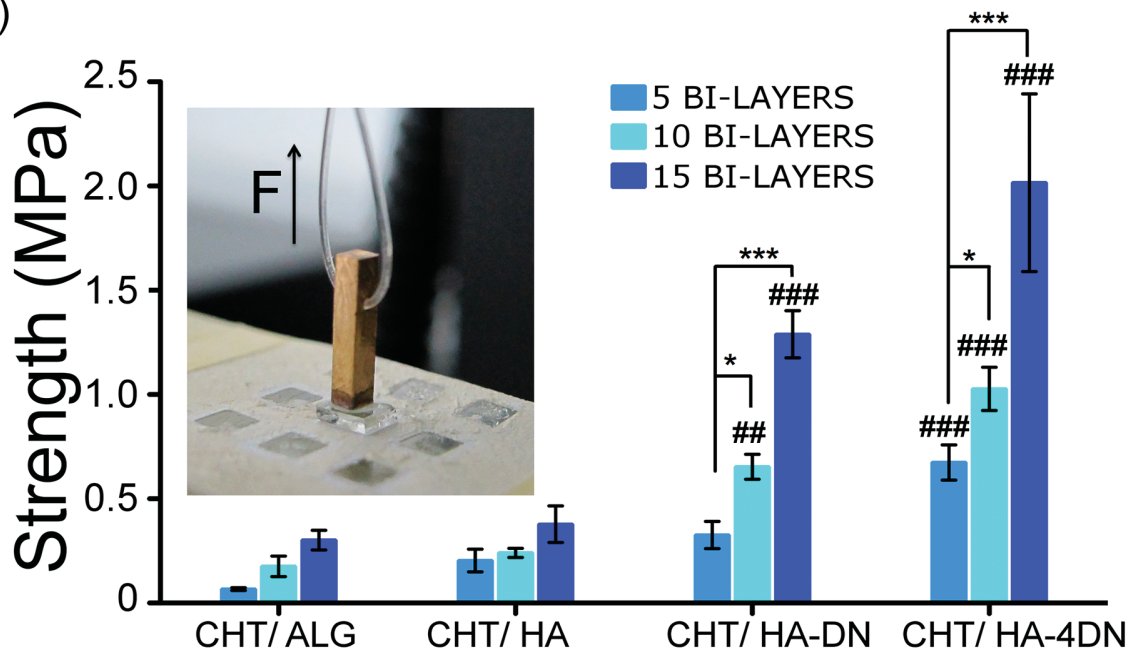

Figure 4. a) Representative force versus displacement curves for the pull-out experiments over CHT/ HA-4DN, CHT/ HA-DN, CHT/ HA, and CHT/ ALG multilayer films. b) Adhesive strength between the multilayer films produced over the wettable regions of the SH microarray and the iron pillars. Data are means SD $(n=5 ; *=p<0.1$, and $* * *=p<0.001)$. The statistical differences relating to CHT/HA films with the same number of bilayers are represented by doubles symbols (\#\#, $p<0.01$ ) and triple symbols (\#\#\#, $<<0.001)$. Representative image of an adhesion measurement in a single spot where an iron pillar is pulled out with a constant strain rate. 
the films containing DOPA is higher, due to its larger adhesive strength. The lap shear strength of the different multilayer films is shown in Figure 4b. It is clear, that upon increasing the number of multilayered films, the adhesion force increases. In addition, multilayer films containing higher amount of catechol group present higher strength. Worth to mention, the obtained adhesion values for the experiments performed using small-sized surfaces are in agreement with results already reported using centimeter-scale surfaces and conventional tests. ${ }^{[25]}$ For instance, spots with 10 bilayers and a contact area of $4 \mathrm{~mm}^{2}$, achieved values varying between $0.65 \pm 0.06$ and $0.24 \pm 0.02 \mathrm{MPa}$, for CHT/HA-DN and CHT/HA, respectively, in agreement with those previously reported for the same systems using larger surfaces. ${ }^{[25]}$

\subsection{In Vitro Cell Studies}

The biological performance of the multilayers was assessed using two cell types (L929-murine fibroblasts and SaOs-2human osteoblast-like cells), both relevant and standard cell lines commonly used in biomedical studies. All biological characterization was performed using image-based analysis directly on the chip, taking advantage of the transparency of the spots. Chips were prepared with spots containing multilayer films composed by 5, 10, and 15 bilayers and featuring the following characteristics: (i) films ending with the negatively charged polymer without any further modification; (ii) films ended with the negatively charged polymers followed by fibronectin adsorption; (iii) positively charged CHT-ended films followed by fibronectin adsorption and; (iv) positively charged CHT-ended films crosslinked with genipin. As can be immediately deduced, two new components (fibronectin and genipin) have been added for these studies since both tested cells are dependent on the presence of proteins for anchorage and extracellular instructions, which turns the composition of the absorbed layer a key point to modulate cell behavior. ${ }^{[47]}$ Fibronectin is an extracellular matrix glycoprotein that plays crucial role in many cell functions such as adhesion. It contains the tripeptide arginine-glycine-aspartic acid (RGD) which is an integrin cell recognition site and that turns it a good candidate to promote cell adhesion onto biomaterials. ${ }^{[48,49]}$ Genipin is a natural product derived from geniposides and has been extensively investigated in the crosslinking of amine-containing polymers (e.g., chitosan, collagen, or gelatin) as well as in multilayered films. ${ }^{[41,50]}$

In a typical experiment, first multilayer films were locally coated with the crosslinking solution or the protein solution (except in the case of the control substrates). Both L929 and SaOs-2 were then seeded on the above-mentioned multilayer films by simple confined deposition of cells suspension droplets in the different spots. Thus, independently on the nature of the multilayer film any desired combination of cells or cell densities can be tested on individual basis. Cell adhesion was assessed by on-spot image-based analysis after $24 \mathrm{~h}$ in culture. Figure 5 shows representative images of the cells nuclei and stained cytoskeleton revealing their attachment and spreading on the LbL films.
Having a quick look to Figure 5 one can immediately determine that, from a qualitative point of view, there are noticeable differences between the different arrays: (i) cells adhered mostly onto surfaces containing DOPA in their composition; (ii) cells tended to adhere more in films with higher number of layers; and (iii) crosslinking also revealed potential to improve cell attachment. The quantitative percentage of area covered by the adhered cells, obtained from image analysis, is represented in Figure 6. Typically, cells assume distinct morphologies depending on the physicochemical and architectural features of the substrate. ${ }^{[51]}$ The cells seeded on the CHT/HA and CHT/ALG multilayer films presented lower adhesion accompanied by round morphology. The higher surface hydration level and softness, compared with the crosslinked films, may justify such performance decay. ${ }^{[41,45]}$ The presence of DOPA in the films, besides enhancing cell attachment, ${ }^{[27]}$ allowed a cell spreading enhancement along the multilayer films, showing a higher percentage of area covered. The individual cell morphology was also analyzed upon culturing the cells on different multilayer films (Figure 6b). An image-based tool (ImageJ) was used to evaluate both the area covered by each cell and the number of cells adhered in the films. Each fluorescence image was divided into grayscale, and the area occupied by the cytoskeleton was determined. The analysis permitted also to count the total number of cell nuclei per spot. Figure $6 c$ shows the correlation between cell number and the fraction of the area covered by the cells. All formulations have presented suitable cells attachment, but the positive effect of the incorporation of DOPA is evident. Cells are more elongated, and thus, with higher area occupied, and the morphology became more spread and trapezoidal-like shape for DOPA multilayer films covered by genipin and fibronectin. Multilayer films with CHT-ended layers and with preadsorption of fibronectin show higher cell attachment compared with the multilayer films ended with negative charged biopolymers. This could be explained due to the fact that fibronectin has an acidic isoelectric point, presenting a negative net charge above $\mathrm{pH}$ 5-6, which would enhance their electrostatic binding to the positive terminal multilayers rather than to the negative ones. Some studies have suggested surfaces containing positively charged functional groups to enhance cell attachment and spreading. ${ }^{[52]}$ The physicochemical properties of the multilayer films are also reported to influence the cell attachment process. ${ }^{[41,53-56]}$ Both cell types have shown similar behavior on the crosslinked multilayers, relatively to the controls (unmodified multilayers). The crosslinking process decreases water content and increases stiffness in multilayers. ${ }^{[41,55]}$ Consequently, L929 and SaOs-2 attachment tended to increase for the multilayers crosslinked with genipin. This observation corroborates previous studies where a similar trend was reported with different cell types. ${ }^{[40,41,57-59]}$ Previously, we evidenced the suitability of such platform for microtissues formation and drug screening, ${ }^{[60,61]}$ as well as, high-content in vivo studies. ${ }^{[62]}$ This work clearly demonstrated that the technology could be extended to the screening of more complex biomaterials (in this case nanostructured multilayers) and to other physicochemical analysis, such as topography, wettability, and adhesion. 
a) $\mathbf{L} \mathbf{9 2 9}$

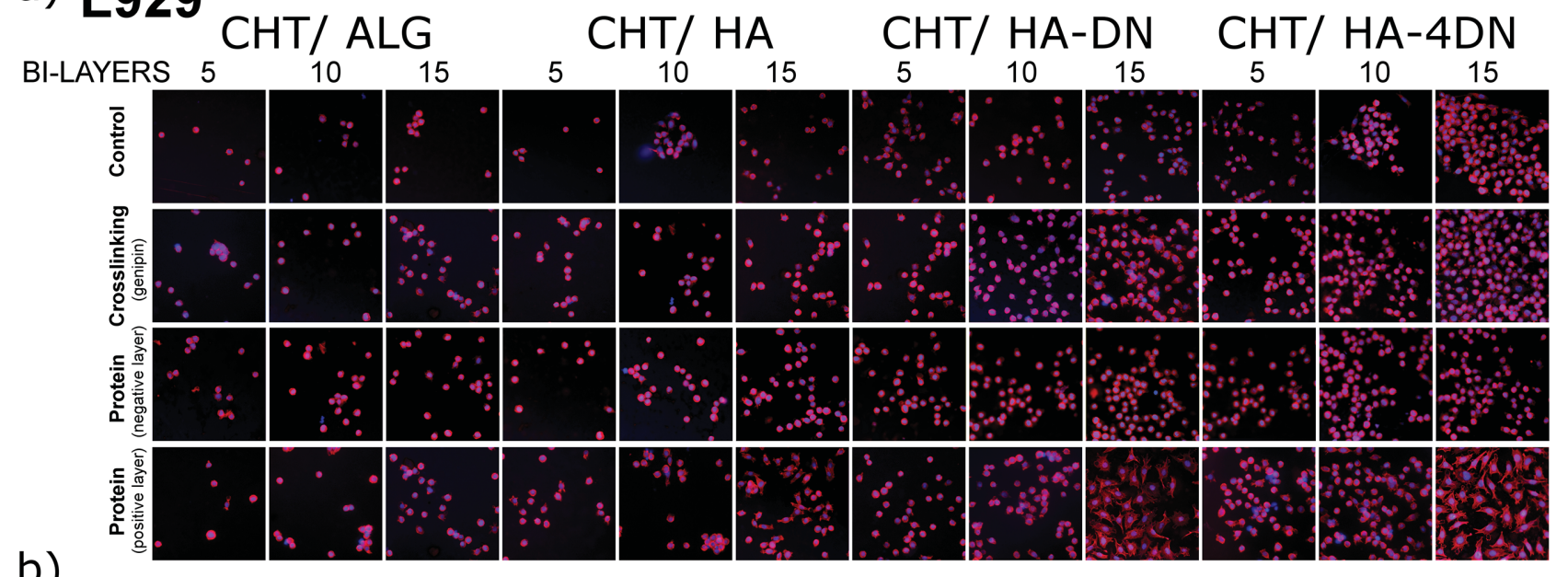

b)

\section{SaOs-2}
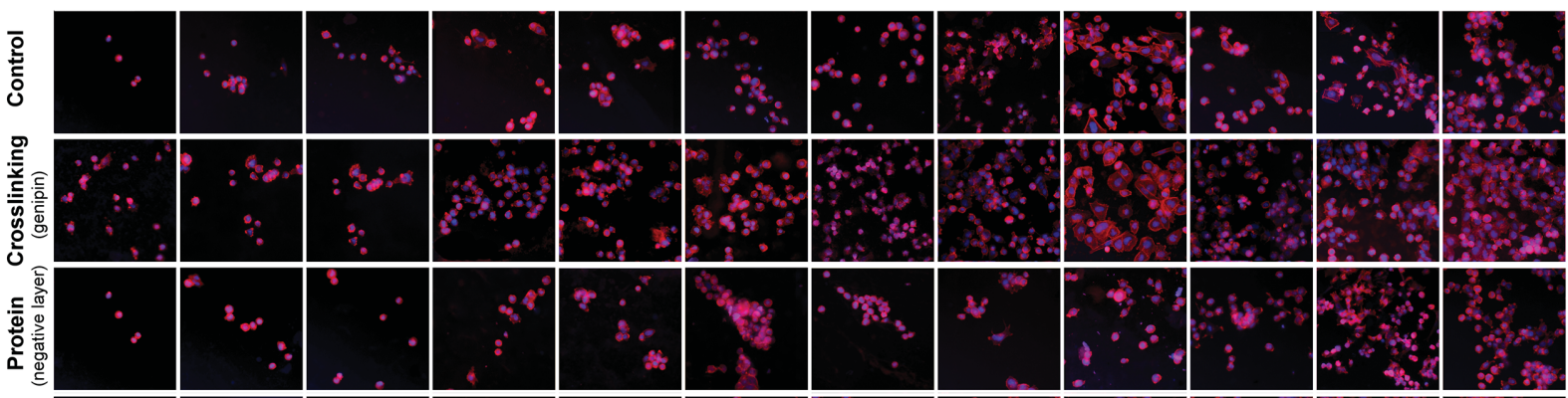

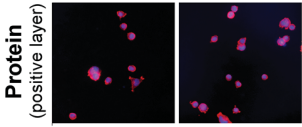
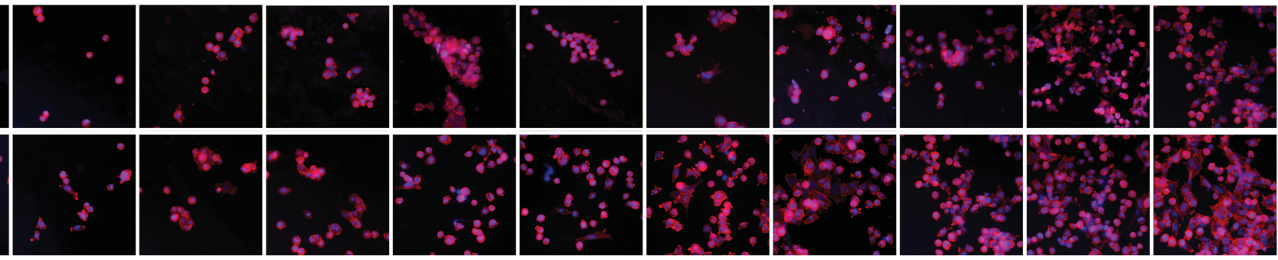

Figure 5. Representative fluorescence microscope images of the coated spots with a) $\mathrm{L} 929$ and b) SaOs-2 cells stained with DAPI/ phalloidin after being cultured for $24 \mathrm{~h}$. Multilayer films of CHT/ALG, CHT/HA, CHT/HA-DM, and CHT/HA-4DN with 5, 10, and 15 bilayers were tested over the patterned chip. Cells attachment was also evaluated in the multilayer patterns coated with fibronectin (over positively or negatively terminating multilayers) or crosslinked with genipin. Scale bar: $50 \mu \mathrm{m}$.

\section{Conclusion}

The preparation of discrete and structured multilayered regions deposited on a single chip that allow for a fast HTS screening has been reported. The platform presents several advantages in comparison with other microarrays already described: (i) The size and composition of the platform, as well as, the geometry of the wettable spots, can be easily controlled in an individual form, and the assays in the liquid state occur only in the desired wettable area without crosstalking between spots; (ii) the SH platform is solely physically modified polystyrene which guarantees the lack of cytotoxicity and the chemical stability of the platform; (iii) the use of microsize droplets for the production of nanostructure films and biological assays allows a drastic reduction of the volume in which the cells are grown; cell culture media can be changed easily without destroying the multilayer films; (iv) drugs or other molecules can be added at any time, and if precautions are taken the mechanical impact in the cells is reduced; (v) the platform is compatible with or easily adapted to many characterization can be performed using image-based analysis directly on the chip taking the benefit of the transparency of the spots and; (vi) high-throughput combinatorial analysis may be performed in situ using different cell types.

As a proof-of-concept for the feasibility of this new approach, we have demonstrated the influence of doping biopolymers such as HA with functional dopamine molecules on three specific LbL properties of interest: (1) final roughness, wettability, and morphology; (2) discrete measurement of adhesion properties of the films; and (3) in situ examination of cells adhesion. Overall, multilayer films containing catechol groups presented higher mechanical adhesion strength and enhanced cell adhesion, showing potential to be used as a new generation of wound healing bandages or surgical sealants, or even, as surface coatings for optimized cell-substrate interaction.

According with the results obtained, we do believe that this versatile and cost-effective platform for enabling HTS may also have potential in several other biomedical areas, including tissue 
a)
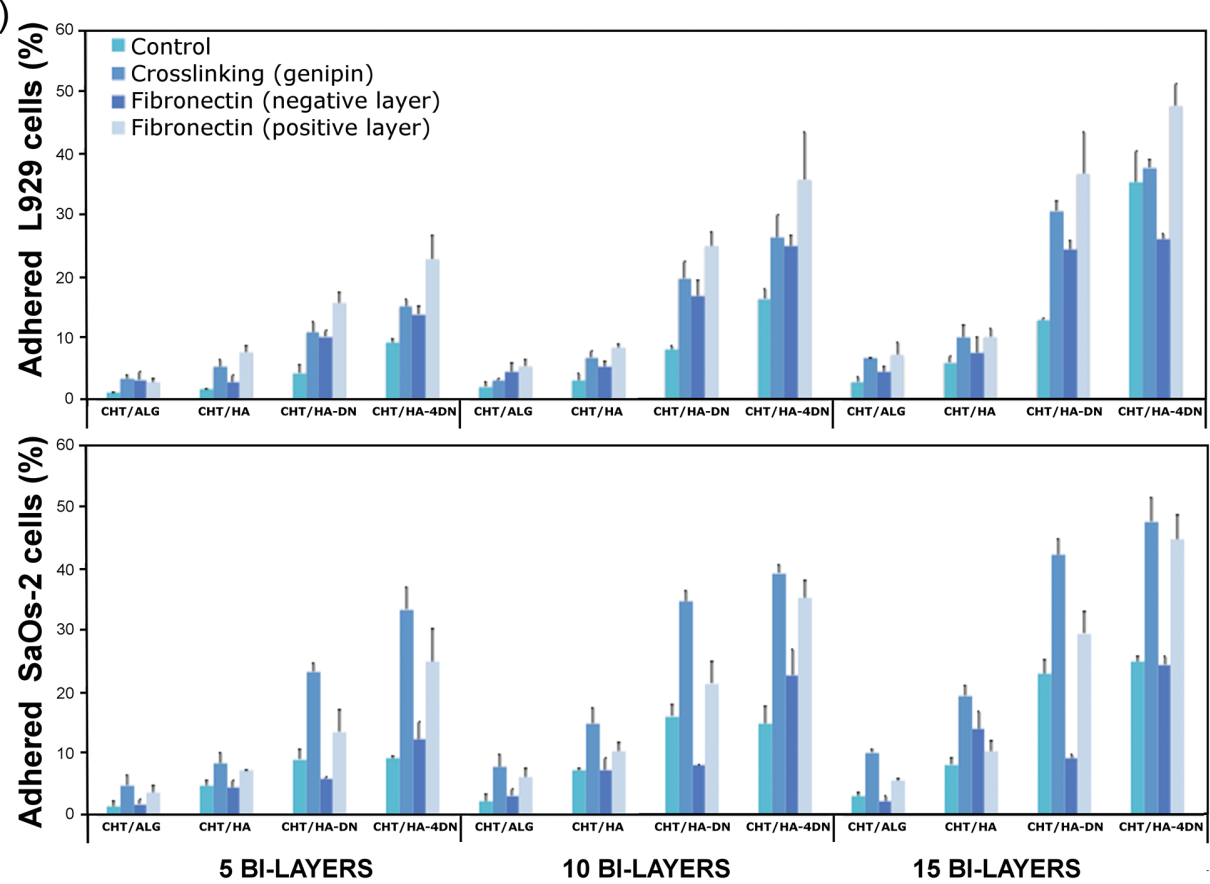

b)

C)
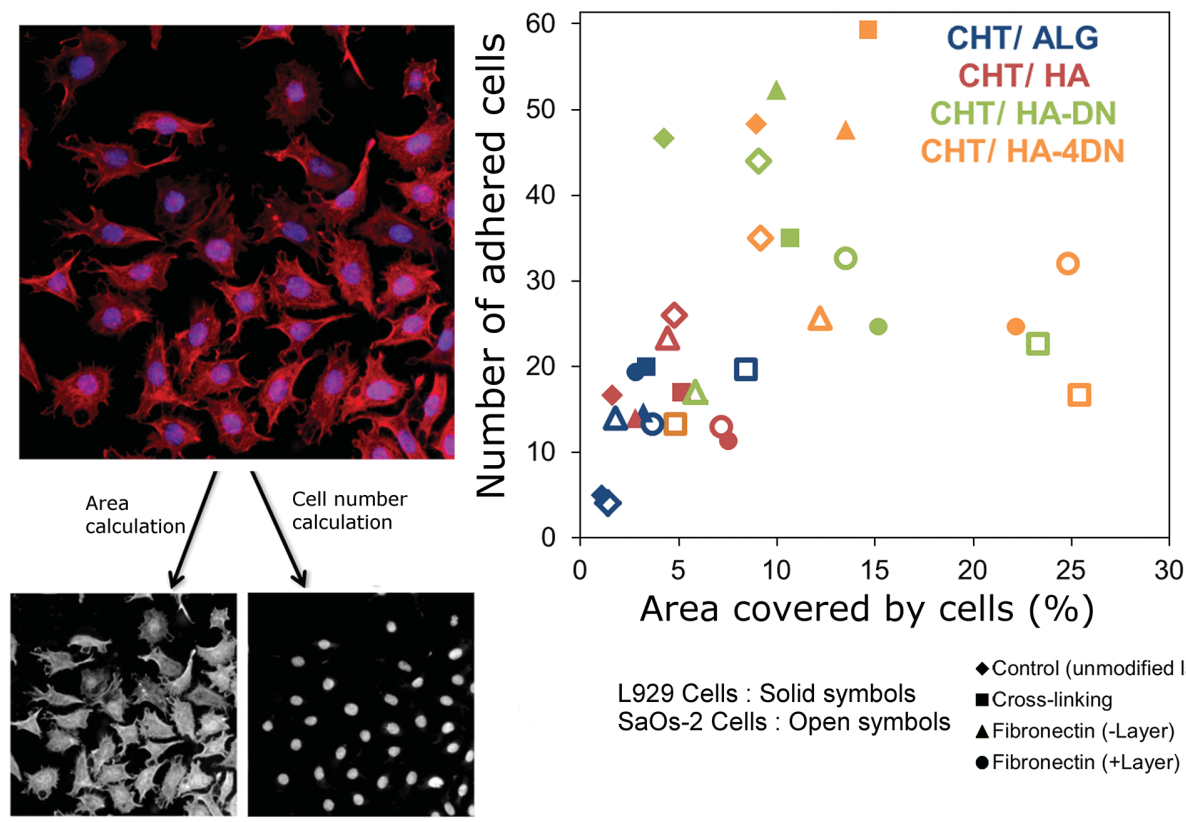

L929 Cells : Solid symbols

- Control (unmodified layers)

SaOs-2 Cells : Open symbols

-Cross-linking

$\Delta$ Fibronectin (-Layer)

- Fibronectin (+Layer)

Figure 6. a) L929 and SaOs-2 cellular density (percent of the area covered by the cells) quantified using image analysis in the studied multilayer films for $(n=3)$. b) Example of an original image, where the number of cells and cell density (percent of the area covered by the cells) were quantified by image analysis; this procedure was performed in all studied multilayer films for L929 and SaOs-2 cells. c) Correlation between the number of cells adhered per spot and density (percentage of the area covered) of L929 cells (solid symbols) and SaOs-2 cells (open symbols). Cells attachment was studied over five bilayer films of CHT/ALC (blue color), CHT/HA (red color), CHT/HA-DN (green color), and CHT/HA-4DN (orange color), also coated with fibronectin and crosslinked with genipin. Scale bar: $50 \mu \mathrm{m}$.

engineering, regenerative medicine and drug discovery, among others. Moreover, this method can be operated as a single lab bench-top apparatus or combined with liquid-handling robotics to extend the library size, allowing this user-friendly platform to achieve considerable advances for miniaturized bioanalytical and biomedical analysis in the near future.

\section{Experimental Section}

Materials: Medium molecular weight $\mathrm{CHT}$ was purchased from SigmaAldrich (ref. 448877, Brookshield viscosity 200-800 cP, molecular weight $190-310 \mathrm{kDa}$ ) and purified by a series of filtering steps and precipitation in water and ethanol, followed by freeze-drying. HA-hyaluronic acid sodium salt from Streptococcus equi-was purchased from Sigma 
(ref. 53747, molecular weight of $595 \mathrm{kDa}$ ). Dopamine hydrochloride (DN) (ref. H8502) and $N$-(3-dimethylaminopropyl)- $N$ '-ethylcarbodiimide hydrochloride (EDC) (ref. 03450, purum, $\geq 98.0 \%$ (AT)) were purchased from Sigma and used without any further purification. ALG-alginic acid sodium salt from brown algae-was purchased from Fluka (ref. 71238 , molecular weight 100-200 kDa). Genipin was purchased from Wako chemical (ref. 078-03021). Human plasma fibronectin (HFN) was purchased from Sigma-Aldrich (95\% pure, 0.1\%). Tetrahydrofuran (THF) was from Fluka (p.a. > 99.5\%) and ethanol absolute from Panreac. Smooth PS sheets were purchased from Goodfellow, UK, with $0.19 \mathrm{~mm}$ of thickness, ref. ST311190/1.

Synthesis of HA-DN: HA-DN conjugates were synthesized using EDC as an activation agent of the carboxyl groups on HA chains, based on the procedure proposed by Lee and co-workers. ${ }^{[44]}$ Briefly, $1 \mathrm{~g}$ of HA was dissolved in $100 \mathrm{~mL}$ of phosphate buffered saline (PBS) solution and the $\mathrm{pH}$ was adjusted to 5.5 with an hydrochloric acid $(\mathrm{HCl})$ aqueous solution. The solution was purged with nitrogen for $30 \mathrm{~min}$. Then, $338 \mathrm{mg}$ of EDC and $474 \mathrm{mg}$ of DN were added and the $\mathrm{pH}$ of the reaction solution was maintained at 5.5 for $2 \mathrm{~h}$. Unreacted chemicals and urea byproducts were removed by extensive dialysis, and afterward the conjugate was lyophilized. In order to avoid oxidation, the conjugate was stored at $4{ }^{\circ} \mathrm{C}$ in a desiccator under vacuum and protected from the light.

Ultraviolet (UV) Spectrophotometry and Quartz Crystal Microbalance (QCM-D): The degree of substitution of dopamine in the conjugate was determined using a UV-vis spectrophotometer (Shimadzu UV-2501 PC) and $1 \mathrm{~cm}$ quartz cells. A solution of $1 \mathrm{mg} \mathrm{mL}^{-1}$ in $0.15 \mathrm{M}$ sodium chloride $(\mathrm{NaCl})$ was prepared for the UV analysis. A Q-sense E4 quartz-crystal microbalance ( $Q$-sense $A B$, Sweden) with a dissipation monitoring system was used to monitor the adsorption of CHT-DN, CHT-4DN, CHT-HA, and CHT-ALG, onto gold-coated quartz crystal in situ. The crystals were previously cleaned with sequential sonication for $3 \mathrm{~min}$ in acetone, ethanol, and isopropanol and then dried with flowing nitrogen gas avoiding contamination prior to use. In order to ensure that the crystals are perfectly clean and therefore show a null frequency, all the experiments started with a $\mathrm{NaCl}$ baseline. The quartz crystal was excited at multiple overtones: 1, 3, 5, 7, 9, 11, and 13, corresponding, respectively, to $5,15,25,35,45,55$, and $65 \mathrm{MHz}$. Adsorption took place at $25^{\circ} \mathrm{C}$ using solutions at $0.5 \mathrm{mg} \mathrm{mL}^{-1}, 0.15 \mathrm{M} \mathrm{NaCl}$ and at a constant flow rate of $100 \mathrm{~mL} \mathrm{~min}{ }^{-1}$, with an adsorption time of $10 \mathrm{~min}$ (starting with $\mathrm{CHT}$ ) and an intermediate rinsing step of $10 \mathrm{~min}$. The frequency of each overtone was normalized to the fundamental resonant frequency of the quartz crystal, by dividing it by $v$ (where $v$ is the number of the overtone, $v=1,3,5, \ldots)$. If a rigid mass is adsorbed onto the surface of the piezoelectric crystal there will be a decrease in the oscillation frequency. For viscoelastic materials, the adsorption of mass does not fully couple to the oscillation of the crystal and dampens the oscillation. QCM-D allows simultaneously measuring the changes in the resonant frequency and in the viscoelastic properties (dissipation) when a film is adsorbed at the crystal surface. CHT was used as the polycation (always the first deposited layer) while HA-DN, HA-4DN, HA, and ALC acted as the polyanion (used as ended-polymer layer). The multilayer systems were assembled at $\mathrm{pH}$ 5.5. The $\mathrm{pH}$ was adjusted with $\mathrm{HCl}$ or $\mathrm{NaOH}$. Films with 10 layers were produced.

Fabrication of Patterned SH Surfaces: Small portions of PS were cut from commercially available smooth PS sheets. Polyvinyl chloride (PVC) stickers (Oracal, USA) were glued into PS surface in the form of an array of $4 \mathrm{~mm}^{2}$ squares separated by $2 \mathrm{~mm}$. The PS surfaces containing square-shaped stickers were modified according to a phase separation protocol described elsewhere. ${ }^{[42]}$ Briefly, superhydrophobic surfaces were produced by dissolving $70 \% \mathrm{v} / \mathrm{v}$ solution of PS (Styrolution PS $158 \mathrm{~K}$ ) in tetrahydrofuran solvent for approx. $2 \mathrm{~h}$ to form a presolution. Then, $1.4 \mathrm{~mL}$ of ethanol was added into $2 \mathrm{~mL}$ of PS presolution and the mixed solution was stirred. A necessary volume to cover the PS substrate with this mixture was dipped onto a cleaned PS smooth surface. After several seconds in air, the substrate with the mixture was immersed in ethanol for $1 \mathrm{~min}$ in order to force the polymer precipitation, leading to the formation of a rough and porous topography at both the nano- and microscale levels. Afterward, the surface was dried at ambient temperature. The stickers were then removed from the surface and the protected regions remained untreated and, consequently, were kept wettable and transparent (see Figure 1a). Prior material deposition, the wettable polystyrene spots were exposed to UV-ozone irradiation (procleaner 220, BioForce Nanoscience), in order to modify chemically the spots incorporating negative charged groups.

Preparation of the Multilayer Films: Multilayer films were constructed manually in the wettable spots of the patterned superhydrophobic surfaces, by pipetting alternately droplets of $5 \mu \mathrm{L}$ of the polyelectrolyte solutions during $10 \mathrm{~min}$, beginning with $\mathrm{CHT}$. The droplets were removed using a pipette, and then, a rinsing step of $5 \mathrm{~min}$ with droplets of $0.15 \mathrm{M} \mathrm{NaCl}$ solution was included between the adsorptions of each polyelectrolyte.

On-Chip Surface Characterization: The surface morphology of the multilayered film on the chip was observed using a Leica Cambridge S-360 SEM. All samples were coated with a conductive layer of sputtered gold. The SEM micrographs were taken at an accelerating voltage of $15 \mathrm{kV}$ and at different magnifications.

The surface roughness of the samples was analyzed by AFM. AFM measurements were performed in a MultiMode STM microscope controlled by the NanoScope III from Digital Instruments system, operating in tapping mode at a frequency of $1 \mathrm{~Hz}$. At least five measurements were performed in the different sample, which had been previously air-dried.

Water contact angle measurements were also performed at room temperature using an OCA 15plus goniometer (DataPhysics Instruments, Germany). The values were obtained by the sessile drop method. The used liquid was ultrapure water and the drop volume was $3 \mu \mathrm{L}$. This volume was small enough so that the droplets could occupy an area lower than the area of the multilayers in the spots. At least five measurements were carried out for each sample. Films with 5,10 , and 15 bilayers were produced onto the wettable spots of the $\mathrm{SH}$ chip. For each multilayer film construction, an array of 15 combinations, containing 5, 10, and 15 bilayers of the same biomaterials was designed (five replicates per combination were used).

Adhesion Tests: The adhesion properties of the multilayer films was evaluated using a universal mechanical testing machine (Instron mode 5540, USA), following the ASTM D1002 standard. Fresh polyelectrolyte solutions were prepared by dissolution of HA-4DN, HA-DN, HA, and ALG in $0.15 \mathrm{M}$ of $\mathrm{NaCl}$ solution and $\mathrm{CHT}$ in $1 \%$ acetic acid, with a $\mathrm{pH} 5.5$ to yield a final concentration of $0.5 \mathrm{mg} \mathrm{mL}^{-1}$.

Films with 5,10 , and 15 bilayers were produced onto the wettable spots of the $\mathrm{SH}$ chip. For each multilayer film construction, an array of 15 combinations, containing 5, 10, and 15 bilayers of the same biomaterials was designed (five replicates per combination were used).

All the adhesion experiments were conducted at $25^{\circ} \mathrm{C}$. Iron pillars (base of $2 \times 2 \mathrm{~mm}$ and height of $10 \mathrm{~mm}$ ) with a small square piece of glass (base of $3 \times 3 \mathrm{~mm}^{2}$ ) glued on the bottom surface of each pillar, were put in contact with the multilayer films and were superimposed and maintained at $40{ }^{\circ} \mathrm{C}$ overnight-see scheme in Figure 1. The small glasses were used to guarantee that the whole nanofilm created is in contact and adhered in the smooth glass surface. Then, the bonded multilayer films and the pillars were placed on the testing machine and tensile tests were individually performed using a crosshead speed of $5 \mathrm{~mm} \mathrm{~min}{ }^{-1}$ until detachment. All iron pillars had a hole on the top, where a "fishing wire" was passed by, in order to pull the pillars. The pillar could be made of other materials or the base could be from any other substance. The lap shear bonding strength was then determined from the maximum of the force-deformation curve obtained. The average and standard deviations were determined using the results from five samples.

Statistical analysis was performed to analyze significant differences between formulations, using two-way analysis of variance (ANOVA) with Bonferroni post-test using GraphPad Prism 5.0 software. The adopted nomenclature was the following: statistical differences in grouped by time point analysis were marked with $(*)$, and $(* * *)$, which stand for $p<0.05$ and $p<0.001$, respectively, and the statistical differences 
relating to $\mathrm{CHT} / \mathrm{HA}$ films with the same number of bilayers were marked by doubles symbols (\#\#, $p<0.01$ ) and triple symbols (\#\#\#, $p<0.001$ ).

Evaluation of Cell Behavior. Two cell lines, namely, a mouse lung fibroblastic cell line (L929) and a human primary osteosarcoma cell line (SaOs-2), were obtained from European Collection of cell Cultures (ECA CC, UK). The cells were cultured with low glucose DMEM supplemented with $3.7 \mathrm{mg} \mathrm{mL}^{-1}$ sodium bicarbonate, $10 \% \mathrm{FBS}$, and $1 \%$ penicillin-streptomycin at $\mathrm{pH}$ 7.4. The cells were grown in $75 \mathrm{~cm}^{2}$ tissue culture flasks and incubated at $37^{\circ} \mathrm{C}$ in a humidified air atmosphere of $5 \% \mathrm{CO}_{2}$. The medium was changed every $3-4 \mathrm{~d}$. At $90 \%$ of confluence, cells grown in tissue culture flasks were washed with PBS and subsequently detached by a chemical procedure with $0.05 \%$ trypsin-EDTA solution for $5 \mathrm{~min}$ at $37^{\circ} \mathrm{C}$ in a humidified air atmosphere of $5 \% \mathrm{CO}_{2}$. To inactivate the trypsin effect, cell culture medium was added. The cells were then centrifuged at $300 \mathrm{~g}$ and $25^{\circ} \mathrm{C}$ for $5 \mathrm{~min}$ and the medium was decanted.

Prior to cell seeding, the multilayer films with 5,10 , and 15 bilayers were built on the patterned superhydrophobic surface. After drying, droplets of genipin and fibronectin were dispensed atop of the created films. An array of 48 combinations of biomaterials based in an initial library of five polyelectrolytes was created. The design of the patterned chip is based on the construction of four parameters of each multilayer film, containing six replicates of the wettable spots with the same number of multilayers deposited. Since two cell types were studied in the same chip, a total of 48 distinct conditions for the same number film layers were used. Based on previous works, genipin was used in the concentration of $1 \mathrm{mg} \mathrm{mL}^{-1},{ }^{[4]]}$ and the patterned slide was slide was incubated for $24 \mathrm{~h}$, in the incubator at $37^{\circ} \mathrm{C}$. After $23 \mathrm{~h}$ of incubation, droplets of fibronectin

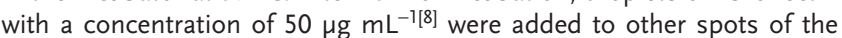
same patterned chip. Afterward, the chip was again incubated for $1 \mathrm{~h}$. The coated films with 5, 10, and 15 bilayers were carefully washed, and sterilized by UV radiation for $30 \mathrm{~min}$. Droplets of $5 \mu \mathrm{L}$ of supplemented DMEM containing a cell suspension with $5 \times 10^{3}$ cells was added on the top of the coated multilayer films. Then, the samples were incubated at $37^{\circ} \mathrm{C}$ in a humidified air atmosphere of $5 \% \mathrm{CO}_{2}$.

After $1 \mathrm{~d}$ in culture, and prior to cell staining, the entire patterned chip was rinsed thrice with sterile PBS and fixed with formalin $2.5 \%(\mathrm{v} / \mathrm{v})$ for cytoskeleton and nuclei staining with phalloidin-tetramethylrhodamine B isothiocyanate (Phalloidin-TRITC, Sigma-Aldrich) and 4,6-diamino-2phenyindole dilactate (DAPI, Sigma-Aldrich). Samples were incubated in the dark with Phalloidin-TRITC solutions $(1: 100)$ for $30 \mathrm{~min}$, then with DAPI for $10 \mathrm{~min}$ (1:1000). After rinsing the samples, the cytoskeleton and nuclei were observed using an Imager Z1 fluorescence microscope (Zeiss) and photographed using an Axio Cam MRm (Zeiss). Image) software was used to calculate the percentage of the area covered by the cells adhered on the multilayer films.

\section{Acknowledgements}

The authors acknowledge the financial support from the FCT (Fundação para a Ciência e para a Tecnologia) through the Ph.D. grants with the references SFRH/BD/73119/2010 and SFRH/BD/70107/2010. The research was also funded by FEDER through the Competitive Factors Operation Program (COMPETE) and by National Funds through FCT in the scope of the project PTDC/CTM-BIO/1814/2012.

Received: November 24, 2015 Revised: December 6, 2015 Published online:

[1] Y. Dzenis, Science 2008, 319, 419

[2] Y. Huang, X. F. Duan, Q. Q. Wei, C. M. Lieber, Science 2001, 291, 630.

[3] B. D. Gates, Q. B. Xu, M. Stewart, D. Ryan, C. G. Willson, G. M. Whitesides, Chem. Rev. 2005, 105, 1171.
[4] R. R. Costa, J. F. Mano, Chem. Soc. Rev. 2014, 43, 3453.

[5] J. F. Quinn, A. P. R. Johnston, G. K. Such, A. N. Zelikin, F. Caruso, Chem. Soc. Rev. 2007, 36, 707.

[6] K. Ariga, J. P. Hill, M. V. Lee, A. Vinu, R. Charvet, S. Acharya, Sci. Technol. Adv. Mater. 2008, 9, 014109.

[7] A. L. Hook, D. G. Anderson, R. Langer, P. Williams, M. C. Davies, M. R. Alexander, Biomaterials 2010, 31, 187.

[8] A. I. Neto, C. A. Custodio, W. Song, J. F. Mano, Soft Matter 2011, 7, 4147.

[9] R. Potyrailo, K. Rajan, K. Stoewe, I. Takeuchi, B. Chisholm, H. Lam, ACS Comb. Sci. 2011, 13, 579.

[10] C. G. Simon Jr., S. Lin-Gibson, Adv. Mater. 2011, 23, 369.

[11] M. B. Oliveira, J. F. Mano, Trends Biotechnol. 2014, 32, 627.

[12] J. Park, P. T. Hammond, Adv. Mater. 2004, 16, 520.

[13] C. Kantak, S. Beyer, L. Yobas, T. Bansal, D. Trau, Lab Chip 2011, 11, 1030.

[14] Y. Wang, Y. Liu, Y. Cheng, E. Kim, G. W. Rubloff, W. E. Bentley, G. F. Payne, Adv. Mater. 2011, 23, 5817.

[15] S. A. Sukhishvili, S. Granick, Macromolecules 2002, 35, 301.

[16] G. K. Such, A. P. R. Johnston, F. Caruso, Chem. Soc. Rev. 2011, 40, 19.

[17] M. Marchl, M. Edler, A. Haase, A. Fian, G. Trimmel, T. Griesser, B. Stadlober, E. Zojer, Adv. Mater. 2010, 22, 5361.

[18] M. Sailer, C. J. Barrett, Macromolecules 2012, 45, 5704.

[19] S. A. Castleberry, W. Li, D. Deng, S. Mayner, P. T. Hammond, ACS Nano 2014, 8, 6580

[20] Josep Sedó, Javier Saiz-Poseu, Felix Busqué, D. Ruiz-Molina, Adv. Mater. 2012, 25, 653.

[21] L. Zhang, H.-B. Yin, J.-J. Luo, P.-H. Yang, J.-Y. Cai, Chinese J. Anal. Chem. 2013, 41, 534.

[22] X. Zhang, Z. Li, X. Yuan, Z. Cui, X. Yang, Appl. Surf. Sci. 2013, 284, 732.

[23] H. H. Kim, J. B. Park, M. J. Kang, Y. H. Park, Int. J. Biol. Macromol. 2014, 70, 516

[24] S. Yuan, Z. Li, J. Zhao, S. Luan, J. Ma, L. Song, H. Shi, J. Jin, J. Yin, RSC Adv. 2014, 4, 31481.

[25] A. I. Neto, A. C. Cibrao, C. R. Correia, R. R. Carvalho, G. M. Luz, G. G. Ferrer, G. Botelho, C. Picart, N. M. Alves, J. F. Mano, Small 2014, 10, 2459.

[26] C. J. Detzel, A. L. Larkin, P. Rajagopalan, Tissue Eng. Pt. B-Rev. 2011, 17, 101.

[27] J. Mullegger, G. Lepperdinger, Mol. Reprod. Dev. 2002, 61, 312.

[28] Y. Luo, K. R. Kirker, G. D. Prestwich, J. Control. Release 2000, 69, 169.

[29] D. Jiang, J. Liang, P. W. Noble, Ann. Rev. Cell Dev. Biol. 2007, 23, 435.

[30] B. Thierry, F. M. Winnik, Y. Merhi, M. Tabrizian, J. Am. Chem. Soc. 2003, 125, 7494

[31] H. Y. Wang, Y. Sun, B. Tang, Talanta 2002, 57, 899

[32] K. A. Marx, Biomacromolecules 2003, 4, 1099.

[33] H. H. Tonnesen, J. Karlsen, Drug Dev. Ind. Pharm. 2002, 28, 621.

[34] J. F. Mano, G. A. Silva, H. S. Azevedo, P. B. Malafaya, R. A. Sousa, S. S. Silva, L. F. Boesel, J. M. Oliveira, T. C. Santos, A. P. Marques, N. M. Neves, R. L. Reis, J. R. Soc. Interf. 2007, 4, 999.

[35] K. Y. Lee, D. J. Mooney, Prog. Polym. Sci. 2012, 37, 106.

[36] P. Bieker, M. Schoenhoff, Macromolecules 2010, 43, 5052.

[37] N. M. Alves, C. Picart, J. F. Mano, Macromol. Biosci. 2009, 9, 776.

[38] G. V. Martins, E. G. Merino, J. F. Mano, N. M. Alves, Macromol. Biosci. 2010, 10, 1444.

[39] G. V. Martins, J. F. Mano, N. M. Alves, Carbohydr. Polym. 2010, 80, 570.

[40] S. G. Caridade, C. Monge, F. Gilde, T. Boudou, J. F. Mano, C. Picart, Biomacromolecules 2013, 14, 1653.

[41] J. M. Silva, A. R. C. Duarte, S. G. Caridade, C. Picart, R. L. Reis, J. F. Mano, Biomacromolecules 2014, 15, 3817.

[42] N. M. Oliveira, A. I. Neto, W. Song, J. F. Mano, Appl. Phys. Exp. 2010, 3. 
www.MaterialsViews.com

[43] A. I. Neto, H. J. Meredith, C. L. Jenkins, J. J. Wilker, J. F. Mano, RSC Adv. 2013, 3, 9352.

[44] H. Lee, Y. Lee, A. R. Statz, J. Rho, T. G. Park, P. B. Messersmith, Adv. Mater. 2008, 20, 1619.

[45] J. Borges, S. G. Caridade, J. M. Silva, J. F. Mano, Macromol. Rapid Commun. 2015, 36, 405.

[46] N. Thirawong, J. Nunthanid, S. Puttipipatkhachorn, P. Sriamornsak, Eur. J. Pharm. Biopharm. 2007, 67, 132.

[47] S. M. Oliveira, V. E. Santo, M. E. Gomes, R. L. Reis, J. F. Mano, Biomaterials 2015, 48, 56.

[48] E. Ruoslahti, M. Pierschbacher, Science 1987, 238, 491.

[49] C. R. Wittmer, J. A. Phelps, C. M. Lepus, W. M. Saltzman, M. J. Harding, P. R. Van Tassel, Biomaterials 2008, 29, 4082.

[50] J. M. Silva, A. R. C. Duarte, C. A. Custódio, P. Sher, A. I. Neto, A. C. M. Pinho, J. Fonseca, R. L. Reis, J. F. Mano, Adv. Healthc. Mater. 2014, 3, 433.

[51] Natália M. Alves, Iva Pashkuleva, Rui L. Reis, J. F. Mano, Small 2010, 6, 2208

[52] K. Webb, V. Hlady, P. A. Tresco, J. Biomed. Mater. Res. 2000, 49, 362
[53] A. Schneider, G. Francius, R. Obeid, P. Schwinte, J. Hemmerle, B. Frisch, P. Schaaf, J. C. Voegel, B. Senger, C. Picart, Langmuir 2006, 22, 1193.

[54] C. Picart, Curr. Med. Chem. 2008, 15, 685

[55] K. Ren, T. Crouzier, C. Roy, C. Picart, Adv. Funct. Mater. 2008, 18, 1378.

[56] S. M. Oliveira, T. H. Silva, R. L. Reis, J. F. Mano, J. Mater. Chem. B 2013, 1, 4406

[57] S. Yamanlar, S. Sant, T. Boudou, C. Picart, A. Khademhosseini, Biomaterials 2011, 32, 5590.

[58] V. Gribova, R. Auzely-Velty, C. Picart, Chem. Mater. 2012, 24, 854.

[59] S. G. Caridade, C. Monge, J. Almodovar, R. Guillot, J. Lavaud, V. Josserand, J.-L. Coll, J. F. Mano, C. Picart, Acta Biomater. 2015 $15,139$.

[60] M. B. Oliveira, A. I. Neto, C. R. Correia, M. Isabel Rial-Hermida, C. Alvarez-Lorenzo, J. F. Mano, ACS Appl. Mater. Interf. 2014, 6, 9488.

[61] A. I. Neto, C. R. Correia, M. B. Oliveira, M. I. Rial-Hermida, C. Alvarez-Lorenzo, R. L. Reis, J. F. Mano, Biomater. Sci. 2015, 3, 581.

[62] M. B. Oliveira, M. P. Ribeiro, S. P. Miguel, A. I. Neto, P. Coutinho, I. J. Correia, J. F. Mano, Tissue Eng. Pt. C-Methods 2014, 20, 851. 